\title{
Exceptional electrostatic phenomenon in ultrathin nanorods: the terminal $\sigma$-hole
}

\author{
Hengyue $\mathrm{Xu}^{1,3, *}$, Daqin Guan ${ }^{2, *}$
}

${ }^{1}$ State Key Laboratory of Chemical Oncogenomics, Tsinghua Shenzhen International Graduate School, Tsinghua University, Shenzhen, China.

${ }^{2}$ Department of Building and Real Estate, Research Institute for Sustainable Urban Development, The Hong Kong Polytechnic University, Hong Kong, China.

${ }^{3}$ Institute of Biopharmaceutical and Health Engineering, Tsinghua Shenzhen International Graduate School, Tsinghua University, Shenzhen, China.

\section{Corresponding author:}

Hengyue Xu, Email: xuhy21@mails.tsinghua.edu.cn

ORCID iD https://orcid.org/0000-0003-4438-9647

Daqin Guan, Email: daqin.guan@polyu.edu.hk

ORCID iD https://orcid.org/0000-0001-5393-2301

\section{Graphical abstract}

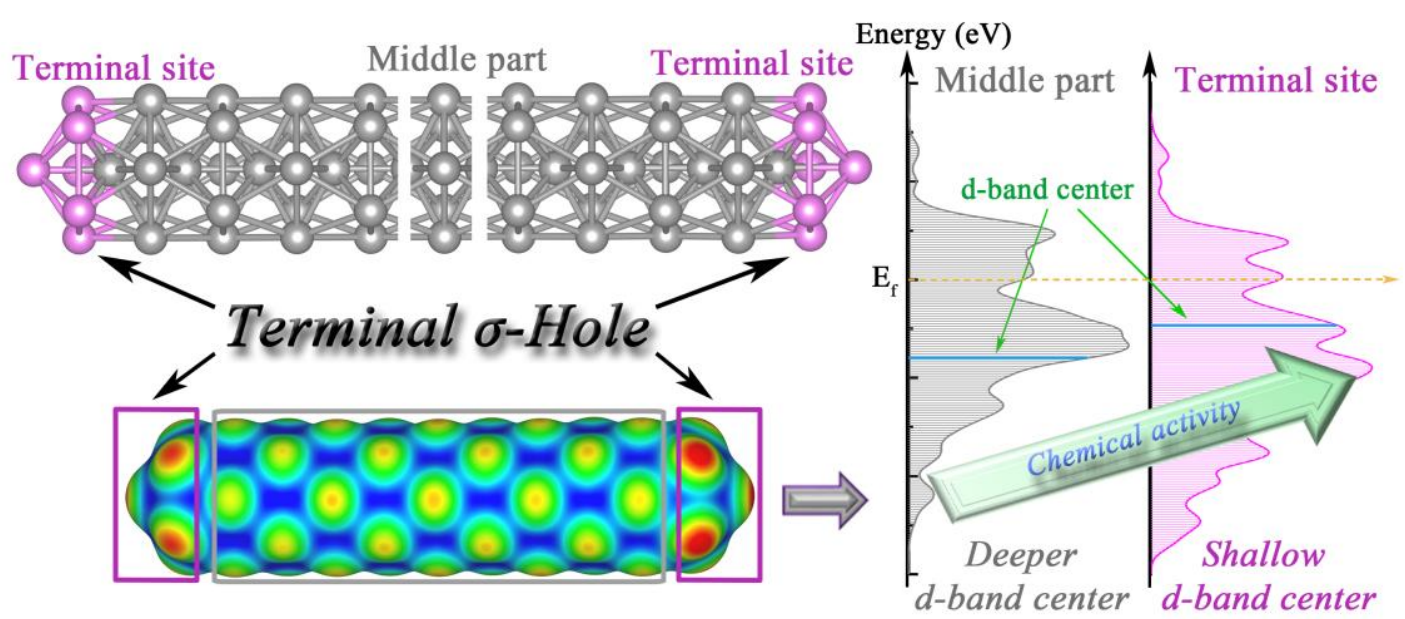




\begin{abstract}
An in-depth understanding of the physicochemical properties of nanorods during the initial growth process has a profound impact on the rational design of high-performance nanorods catalysts. Herein, we conducted a systematic DFT study on the transition metal Co, Ni and alloyed nanoclusters/rods systems to simulate an atomic process from the initial nanoclusters growth to nanorods/wires. We found that the highly active sites of nanorods depend on an interesting electrostatic phenomenon. The surface electrostatic potential analysis shows that all nanoclusters and nanorods structures have formed $\sigma$-hole. Unlike nanoclusters, the $\sigma$-hole only appears at terminal sites in nanorods, called terminal $\sigma$-hole. The elemental composition in nanorods has a certain influence on the maximal surface electrostatic potential $\left(V_{S, \max }\right)$ i.e., terminal $\sigma$-hole. Interestingly, we found that the terminal $\sigma$-hole formed in nanorods is generally higher in magnitude than smaller nanoclusters. First-principle calculations show that terminal $\sigma$-hole is closely related to the physicochemical activities of nanorods. For example, the work function of the directions forming terminal $\sigma$-hole is smaller than other directions. More interestingly, we found that in almost all nanorods, compared with other atoms, the d-orbital of the atoms forming terminal $\sigma$-hole shifts close to the Fermi level and exhibits a shallower d-band center, showing higher chemical activity. In short, it is the first time that we discovered terminal $\sigma$-hole in nanorods, explained the theoretical basis of terminal $\sigma$-hole in nanorod systems, and provided theoretical guidance for the rational design of high-performance nanorods catalysts.
\end{abstract}

Keywords: Co-Ni nanorods, Terminal $\sigma$-hole, First-principle, Growth mechanism, Electrostatic potential.

\title{
INTRODUCTION
}

Nanorods are versatile in the field of catalysis. Their elemental composition, crystal structure and morphology are highly plastic, which can be specifically adjusted to design efficient catalysts for various catalytic reactions ${ }^{1}$. Cobalt and nickel are two 
transition metal elements commonly used in the catalytic industry ${ }^{2-5}$. Compared with precious-metal-based catalysts, cost-effective cobalt and nickel also have rich electronic structural features ${ }^{6,7}$. Therefore, nanoclusters and nanorods composed of Co and $\mathrm{Ni}$ are not only high-quality magnetic materials ${ }^{8,9}$, but also have great potential in catalytic applications. They are widely used in Hydrogen evolution reaction (HER) ${ }^{10-}$ ${ }^{12}, \mathrm{CO}_{2}$ activation $^{13-15}$, Methane activation ${ }^{16}$, etc. However, how to design highly active Co-Ni nanoclusters/rods is still challenging. The theoretical basis behind the catalyticrelated factors, such as the level of chemical activity of nanoclusters/rods and reaction sites is still unclear. Moreover, the current theoretical research on Co-Ni nanoclusters/rods is almost vacant. Therefore, while studying the macroscopic properties of nanorods, it is necessary to conduct DFT-based systematic theoretical researches on geometric structure, energy, charge, magnetism, chemical activity, and catalytic sites of nanorods.

Non-covalent interactions are often the first driver of surface catalysis. From a physical point of view, for a catalyst to catalyze a substrate, it first has to adsorb with the substrate molecules. This interaction often starts from an electrostatically driven noncovalent interaction. At present, $\sigma$-hole as a concept describing many non-covalent forces has been widely used in catalysis ${ }^{17,18} . \sigma$-hole is the area where the electron density decreases due to the anisotropic distribution of the electron density on the atom. When the electron defect reaches a certain level, a positive electrostatic potential region will be generated, which can form an electrostatically driven non-covalent interaction between other molecules with negative electrostatic potential sites ${ }^{19}$. Until recently, $\sigma$ hole was directly observed in experimental imaging ${ }^{20}$. However, in fact, as early as the 2005 conference, Clark et al. conceptualized this phenomenon to solve the unexplainable halogen bonding problem at that time $\mathrm{e}^{21}$. Initially, the condition for forming a $\sigma$-hole was thought to require the halogen half-filled $p$-orbitals to participate in the formation of $\sigma$-orbitals. Since Murray et al. extended the condition of the semifilled $p$-orbital to the $s$-orbital, it is not surprising that the elements which can form a $\sigma$ hole extends from halogen elements to the IV-VI group elements ${ }^{22}$. 
Recently, Stenlid et al. discovered that metals could also use semi-filled $s$-orbitals or $d$ orbitals to form $\sigma$-orbitals so that $\sigma$-hole can also be formed on transition metals ${ }^{23}$. Since then, the $\sigma$-hole concept has been extended to transition metal nanoclusters. Stenlid et al. pointed out that the high chemical activity of metal clusters depends on the proportion of atoms forming $\sigma$-holes, and as the size of nanoclusters increases, the density of corners, which formed $\sigma$-holes decreases ${ }^{24}$. This discovery also explains the phenomena that in early experiments and theoretical reports, the catalytic activity of related nanoclusters will decrease with the increase of the three-dimensional size of nanoclusters ${ }^{25,26}$. So if nanoclusters grow in one dimension, that is, a system of pseudoone-dimensional rod-shaped nanoparticles, is there a similar rule? In many experiments on nanorods, the nanorods used are all very long structures, but they can still show excellent catalytic performance ${ }^{1,27}$. Based on extensive experimental facts, the physicochemical activity (at least some special sites) is preserved in the growth of nanorods.

Based on the above problems, we conducted a systematic DFT study on the different lengths of Co-Ni nanoclusters/rods systems. Our research shows that after nanoclusters grow into nanorods, $\sigma$-hole still exists, but the difference is that $\sigma$-hole only appears on the terminal sites of the nanorods. Furthermore, the terminal $V_{S, \max }$ values higher in magnitude than smaller clusters in all calculated nanorods. We call this phenomenon terminal $\sigma$-hole. Combining geometric structure, energy, charge, electrostatic potential, work function, and density of states analysis, we found that the formation of the terminal $\sigma$-hole will increase the physicochemical activity. After the nanoclusters grow to nanorods, terminal $V_{S, \max }$ values increases, and the d-band center of the terminal-site atoms also moves toward the Fermi level, enhanced the physicochemical activity of terminal sites. The element types of $\mathrm{Co}$ and $\mathrm{Ni}$ have a certain influence on the magnitude of terminal $\sigma$-hole due to the difference in the electron distribution of their atomic orbitals. (Co, $4 \mathrm{~s}^{2} 3 \mathrm{~d}^{7}$, contains 3 semi-filled $d$-orbitals at ground state; $\mathrm{Ni}, 4 \mathrm{~s}^{2} 3 \mathrm{~d}^{8}$, contains 2 semi-filled $d$-orbitals at ground state). The magnitude of terminal $\sigma$-hole, just like other physicochemical properties such as cohesive energy, residual energy, 
magnetic moment, charge distribution, etc., can be adjusted by changing the composition and spatial distribution of elements.

\section{COMPUTATIONAL DETAILS}

\subsection{DFT parameters}

All spin-polarized DFT calculations were performed with the Vienna Ab initio simulation package $\left(\mathrm{VASP}^{28}\right)$. The exchange-correlation function was handled using the generalized gradient approximation (GGA) formulated by the Perdew-BurkeErnzerhof $(\mathrm{PBE})^{29}$. The interaction between the atomic core and electrons was described by the projector augmented wave method ${ }^{30,31}$. The plane-wave basis set energy cutoff was set to $400 \mathrm{eV}$. The spin-polarized calculations were performed starting from default values of NIONS*1.0 as the initial magnetic moment for each atom in the VASP code. All structures with a dynamic magnetic moment were fully relaxed to optimize without any restriction until their total energies were converged to $<10^{-6} \mathrm{eV}$, and the average residual forces were $<0.01 \mathrm{eV} / \AA$. The global transferred charge was calculated by the atomic Bader charge analysis ${ }^{32,33}$.

\subsection{Structural models}

In nanoclusters and nanorods systems, A $1 \times 1 \times 1 \mathrm{k}$-point mesh for the reciprocal space integration was employed for all the calculations with a discrete character. The periodic boundary conditions were implemented with at least $10 \AA$ vacuum to preclude the interaction between a cluster and its image. The simulation boxes were $15 \times 15 \times 15$ for 13 -atoms, $15 \times 15 \times 18 \AA$ for 19 -atoms, $15 \times 15 \times 18 \AA$ for 19 -atoms, $15 \times 15 \times 21 \AA$ for 25 atoms, $15 \times 15 \times 24 \AA$ for 31 -atoms, $15 \times 15 \times 26 \AA$ for 37 -atoms, $15 \times 15 \times 29 \AA$ for 43 -atoms,

$15 \times 15 \times 31 \AA$ for 49 -atoms, $15 \times 15 \times 33 \AA$ for 55 -atoms, $15 \times 15 \times 35 \AA$ for 61 -atoms, $15 \times 15 \times 36 \AA$ for 67 -atoms configurations, respectively. For the nanowire model, all models need to be optimized for the unit cell first, and then a $11 \times 1 \times 1 \mathrm{k}$-point mesh 
for the reciprocal space integration was employed. The simulation boxes were $4.36 \times 15 \times 15$ for periodic 12 -atoms.

\section{RESULTS AND DISCUSSION}

\subsection{Structural features}

According to previous reports, researchers usually observe from experiments that FCCderived nanoparticle geometric structures mainly include truncated octahedrons, cubic octahedrons, and icosahedrons under independent conditions ${ }^{34,35}$. The icosahedral structure is the local geometric minimum within its size range. This model is often used in various theoretical and experimental studies on nanoparticles ${ }^{9,14,36-38}$. We first obtained the 13-atom icosahedral nanocluster A@ B-1 with $I_{h}$ symmetry through spinpolarized DFT calculations. By translating and rotating the six adjacent atoms on the surface of the nanocluster by $36^{\circ}$, an 18-atom short nanorod A@B-2 can be obtained. Continue to translate and rotate $36^{\circ}$ to obtain longer nanorods A@B-C (C=number of core atoms, as shown in Figure 1a, b). We use this operation to build an atomic model to simulate the atomic process growth from nanoclusters to nanorods. After performing this operation indefinitely, we obtain a one-dimensional nanowire A@B-inf with a periodic Z-axis structure (as shown in Figure 1c), simulating the atomic process from nanorods growth to nanowires.

In this study, we mainly studied Co, Ni nanoclusters, nanorods $(<2.5 \mathrm{~nm})$ systems, and their mutual core-shell hybrid structures. Figure 1d and 1e are schematic diagrams of $\mathrm{Co}_{\mathrm{N}}$ and $\mathrm{Ni}_{\mathrm{N}}$ pure metal nanoclusters and nanorods, the number of atoms ranges from 13 to 67 , and the length is about $0.5 \mathrm{~nm}$ to $2.5 \mathrm{~nm}$. Figures $\mathrm{f}$ and $\mathrm{g}$ show the structure of mutual core-shell doping, and there are two configurations: $\mathrm{NiX}_{\mathrm{X}} @ \mathrm{Co}_{\mathrm{N}-\mathrm{X}} 、 \mathrm{Cox}_{\mathrm{X}} @ \mathrm{Ni}_{\mathrm{N}-}$ $\mathrm{X}$ ( $\mathrm{X}$ is the number of core atoms). In the $\mathrm{Nix}_{\mathrm{X}} @ \mathrm{CoN}-\mathrm{x}$ configuration (Figure 1f), Ni atoms are located inside the nanorods, and Co atoms are located on the surface of the nanorods, which have been marked with black arrows in the figure 1f. Replace Co and 
Ni to get the Cox@ $\mathrm{Ni}_{\mathrm{N}-\mathrm{X}}$ configuration, as shown in Figure 1g.

We discovered a series of interesting geometric phenomena in the construction process from nanoclusters to nanorods to nanowires. First, during the process of nanoclusters growing into nanorods $(\mathrm{A} @ \mathrm{~B}-1 \rightarrow \mathrm{A} @ \mathrm{~B}-2)$, the symmetry of the structure changes from $I_{h}(\mathrm{~A} @ \mathrm{~B}-1)$ to $D_{5 h}(\mathrm{~A} @ \mathrm{~B}-1)$. More interestingly, during the process of nanorods growing into nanowires (A@B-2 $\rightarrow \mathrm{A} @ \mathrm{~B}$-inf), the symmetry alternates between $D_{5 h}$ and $D_{5 d}$ (Figures s1, s2), A@B-2 $\left(D_{5 h}\right) \rightarrow \mathrm{A} @ \mathrm{~B}-3\left(D_{5 d}\right) \rightarrow \mathrm{A} @ \mathrm{~B}-4\left(D_{5 h}\right) \rightarrow \mathrm{A} @ \mathrm{~B}-5$ $\left(D_{5 d}\right) \rightarrow \mathrm{A} @ \mathrm{~B}-6\left(D_{5 h}\right) \rightarrow \mathrm{A} @ \mathrm{~B}-7\left(D_{5 d}\right) \rightarrow \mathrm{A} @ \mathrm{~B}-8\left(D_{5 h}\right) \rightarrow \mathrm{A} @ \mathrm{~B}-9\left(D_{5 d}\right) \rightarrow \mathrm{A} @ \mathrm{~B}-$ $10\left(D_{5 h}\right) \rightarrow \rightarrow \rightarrow \mathrm{A} @ \mathrm{~B}-\inf \left(D_{5 h}\right)$.

\subsection{Basic energy and magnetic properties}

The energy property is an important indicator to measure whether a catalyst can exist stably. We first analyzed the change of cohesive energy during the growth of nanorods. The formula for cohesive energy is as follows ${ }^{39}$ :

$$
E_{c o h}=\frac{1}{N}\left(E_{\text {sys }}-n \cdot \sum E_{\text {atom }, x}\right)
$$

$E_{c o h}$ is the cohesive energy per atom, $E_{s y s}$ is the total energy of the nanoclusters/rods structures, and $E_{a t o m, x}$ is the energy of each isolated atom. $x$ is the type of element, $n$ is the number of the $x$ type atoms, and $N$ is the total number of atoms in the nanoclusters/rods structures. We noticed that the cohesive energy of all configuration structures decreases with the growth of nanorods, which means that the growth of nanorods tends to be thermodynamically stable (Figure 1h). For 13-atom icosahedral nanoclusters, the order of cohesive energy is Co@Ni-1 $<$ Co-1 $<\mathrm{Ni}-1<\mathrm{Ni} @ \mathrm{Co}-1$. What is interesting is that when nanoclusters grow into nanorods, the order of their cohesive energy becomes: $\mathrm{Co}-\mathrm{C}<\mathrm{Co} @ \mathrm{Ni}-\mathrm{C}<\mathrm{Ni} @ \mathrm{Co}-\mathrm{C}<\mathrm{Ni}-\mathrm{C}(\mathrm{C}=2 \sim 10$, inf $)$.

Usually, excess energy is one of the critical parameters for studying the relative stability of alloy systems. Here, the excess energy formula ${ }^{5}$ of metal nanoclusters/rods is: 


$$
E_{e x c}=E_{A_{x} @ B_{N-x}}-\frac{x}{N} E_{A_{N}}-\frac{N-x}{N} E_{B_{N}}
$$

Where $E_{\text {exc }}$ is the excess energy of metal nanoclusters/rods, $E_{A_{x} @ B_{N-x}}, E_{A_{N}}, E_{B_{N}}$ are the total energy of the corresponding system, and $A$ and $B$ represent two different elements, $N$ is the total number of atoms in the system. For the pure metal nanoclusters/rods structures, the excess energy is defined as zero unbiased quantity. In the alloyed nanoclusters/rods systems, excess energy can be used as one of its relative stability measures. The negative excess energy $E_{\text {exc }}$ indicates that the structure has high relative stability. Figure $1 \mathrm{i}$ shows the excess energy of all nanoclusters/rods $<2.5 \mathrm{~nm}$. We found that all the excess energy of nanoclusters/rods follows the rule: Co@Ni system $<0$ eV $<\mathrm{Ni@Co} \mathrm{system.} \mathrm{This} \mathrm{excess} \mathrm{energy} \mathrm{rule} \mathrm{indicates} \mathrm{that} \mathrm{Ni}$ atoms tend to segregate to the surface during the growth of Co-Ni nanowires, while Co atoms tend to be enriched in the core. The nanoclusters/rods with the Co core Ni surface are thermodynamically more stable than the Ni core Co surface, which is consistent with the cohesive energy situation. In addition, we also found that with the growth of Co@Ni nanoclusters/rods, the excess energy decreases, which indicates that as the length of the nanowires increases, the $\mathrm{Ni}$ atoms tend to segregate to the surface. However, it is interesting that the excess energy first increases and then decreases in the $\mathrm{Ni@Co} \mathrm{system.} \mathrm{All} \mathrm{the} \mathrm{excess} \mathrm{energy} \mathrm{values} \mathrm{are}>0 \mathrm{eV}$ and reach a maximum value of $3.22 \mathrm{eV}$ at about $2.0 \mathrm{~nm}$, indicating that the growth of $\mathrm{Ni} @$ Co nanorods to about 2.0 $\mathrm{nm}$ has gone through a relatively active thermodynamics stage.

Nano-scale metals have always attracted attention as an interesting magnetic material ${ }^{9,40,41}$. In the Co-Ni metal nanoclusters/rods systems, we also observed that the length and magnetic moment are basically linear in all configurations of the nanoclusters/rods. The order of the magnetic moments of different systems is: pure $\mathrm{Ni}$ $<\mathrm{Co} @ \mathrm{Ni}<\mathrm{Ni} @ \mathrm{Co}<$ pure Co. (For detailed energy and magnetic moment values, see Table S1) 

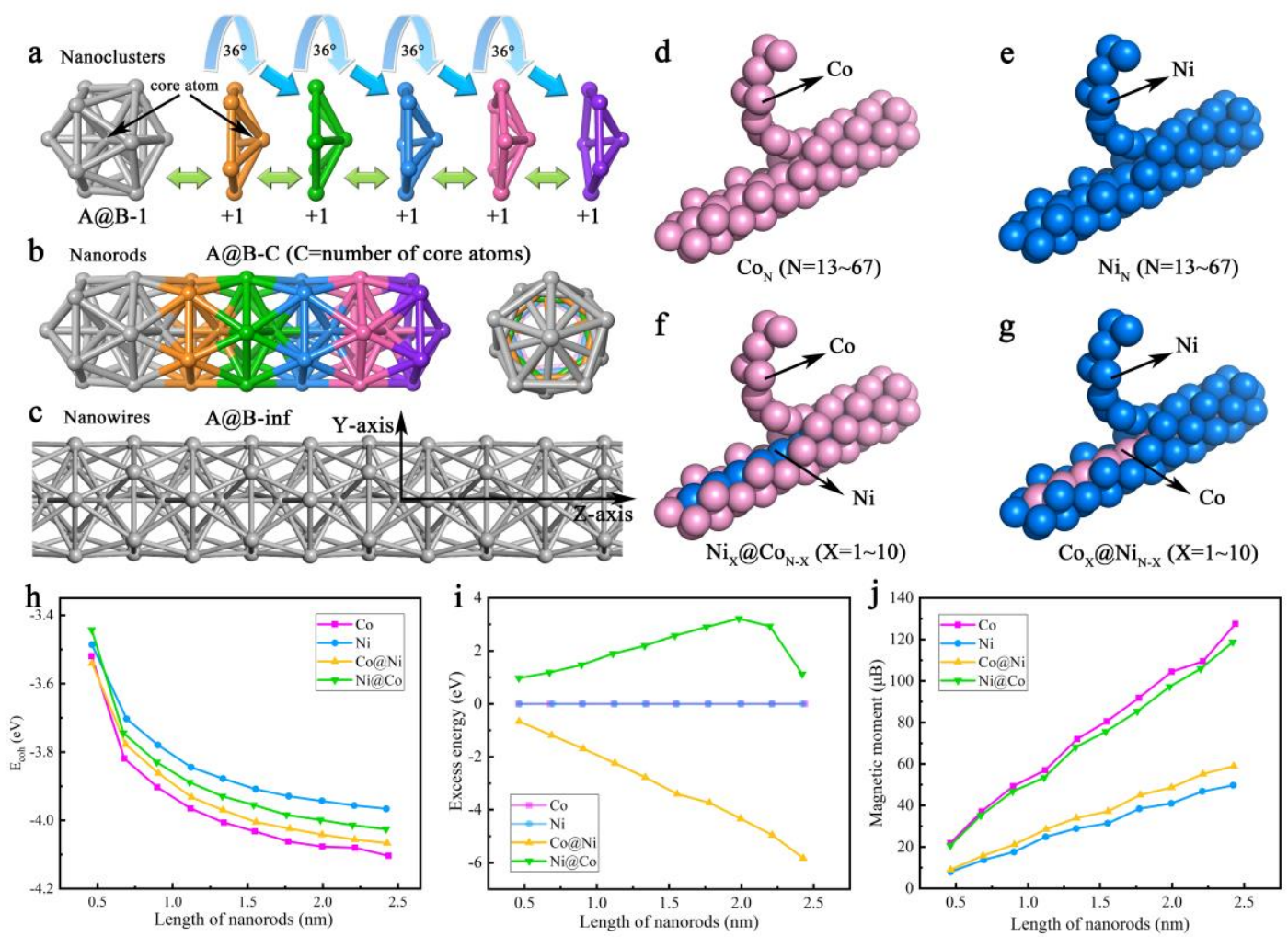

Figure 1. a. The construction of Co-Ni metal nanoclusters/rods/wires model. b. The geometry structures of nanorods. c. The geometry structures of nanowires. d-g. The schematic diagram of structural composition Co-Ni metal nanoclusters/rods (Co in magenta, Ni in blue). h. The cohesive energy. i. The excess energy. j. The magnetic monument. (In figure h-j, Co is represented by a solid magenta line with a rectangle mark in the figure, $\mathrm{Ni}$ is a round blue line, $\mathrm{Co} @ \mathrm{Ni}$ is a yellow positive triangle line, and $\mathrm{Ni@Co} \mathrm{is} \mathrm{a} \mathrm{green} \mathrm{inverted} \mathrm{triangle} \mathrm{line.)}$

\subsection{The terminal $\sigma$-hole concept}

The nanorods structure is widely used in catalysis due to its excellent reactivity. As we all know, chemical reactions often occur on the catalyst's surface, and the surface properties are the critical physicochemical properties of the catalyst. In order to further study the catalytic activity during the growth of the nanorods and the exact location of the catalytic sites on the surface, we calculated the surface electrostatic potential of the Co-Ni nanoclusters/rods. It is defined as follows ${ }^{42}$ : 


$$
V_{E(\boldsymbol{r})}=\sum_{A} \frac{Z_{A}}{\left|\boldsymbol{R}_{A}-\boldsymbol{r}\right|}-\int \frac{\rho\left(\boldsymbol{r}^{\prime}\right) d \boldsymbol{r}}{\left|\boldsymbol{r}^{\prime}-\boldsymbol{r}\right|}
$$

The molecular electrostatic potential $V_{E(\boldsymbol{r})}$ is a mature tool for analyzing chemical bonds and intermolecular interactions. $Z_{A}$ is the charge on the $A$ atom, located at $\boldsymbol{R}_{A}$. $\rho\left(\boldsymbol{r}^{\prime}\right)$ is the charge density. As early as 1992 , Brinck et al. used electrostatic potential to explain the interaction mode of Lewis acid or base with halogen and the formation principle of halogen bond ${ }^{42}$. Later, with the proposal of the $\sigma$-hole concept, the halogen bond can be explained by the $\sigma$-hole ${ }^{21}$. $\sigma$-hole is the region of positive molecular electrostatic potential, widely used to explain non-covalent bond interactions. In recent years, Joakim et al. extended the $\sigma$-hole concept to metal nanoclusters ${ }^{24}$, theoretically explaining the good catalytic activity of gold nanoclusters.

Joakim et al. theoretically explained the increasing catalytic activity of transition metal nanocluster catalysts (such as gold and platinum) as the particle size decreases based on electrostatic potential. In the study of Joakim et al., as the size of the nanoclusters increases, the values of the corner $V_{S, \max }$ are similar in magnitude to those of the smaller nanoclusters ${ }^{24}$. Interestingly, the results of our electrostatic potential calculations show that after nanoclusters grow to nanorods, the $\sigma$-hole still exists at the terminal sites (Figure 2). We call this electrostatic effect in nanorods: the terminal $\sigma$-hole. Excitingly, the magnitude of terminal $\sigma$-hole generally increases after nanoclusters grow into nanorods (Figure 2c), which means that as the nanoclusters grow to nanorods, its terminal sites has a catalytic activity similar to or higher than that of the nanoclusters. (For detailed statistics of electrostatic potential, see Table S2) In addition, the $V_{S, \max }$ of almost all Co surface structures are higher in magnitude than that of Ni surface structures, attributed to Co $\left([\mathrm{Ar}] 4 \mathrm{~s}^{2} 3 \mathrm{~d}^{7}\right)$ having more semi-filled d-orbitals than $\mathrm{Ni}$ $\left([\mathrm{Ar}] 4 \mathrm{~s}^{2} 3 \mathrm{~d}^{8}\right)$ at the ground state. 


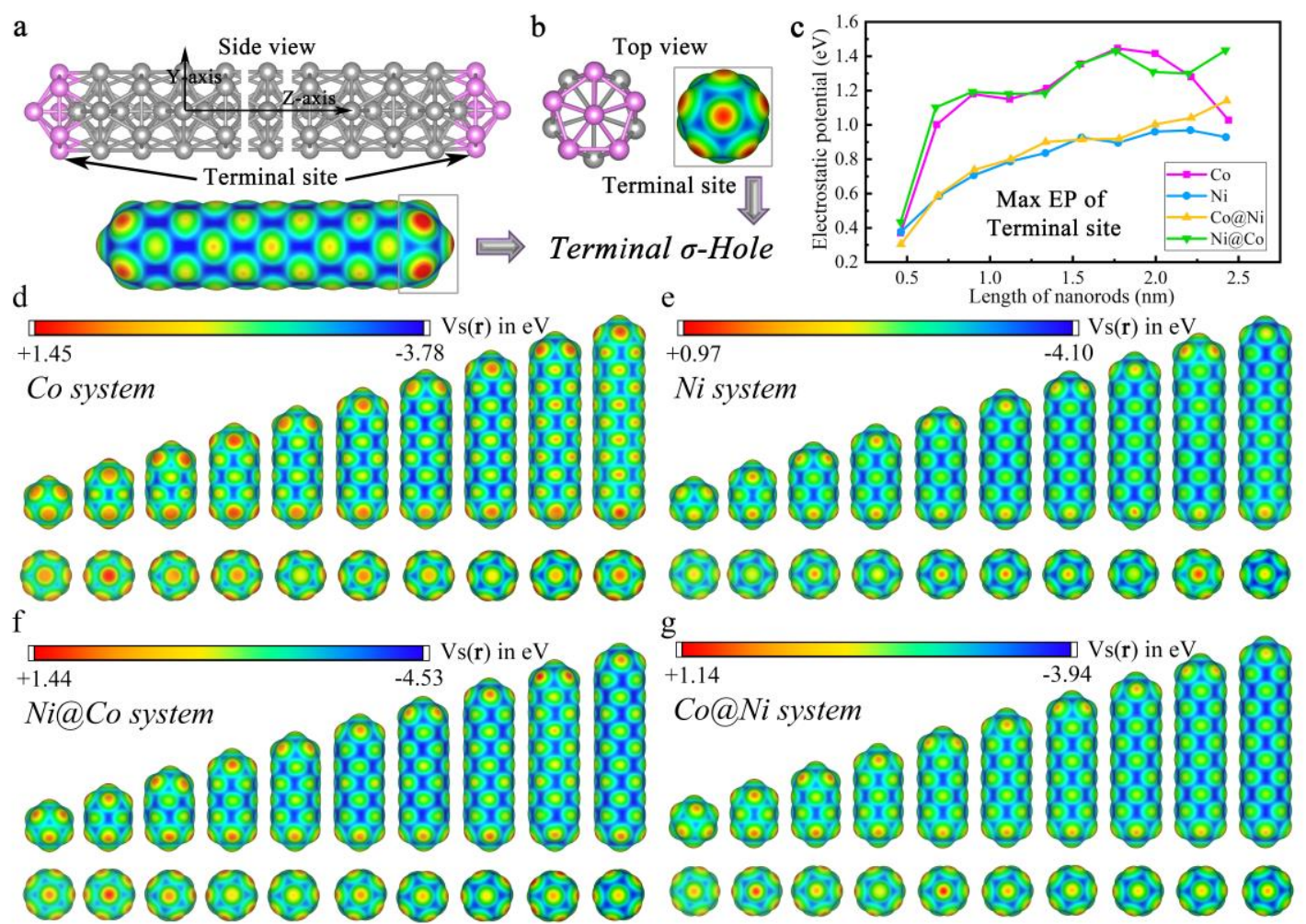

Figure 2. a-b. Side view and top view of nanorods. The terminal sites were highlighted in a grey rectangular frame. c. The statistics of the maximum electrostatic potential of terminal sites in the Co-Ni Nanoclusters/rods growth system. Co is represented by a solid magenta line with a rectangle mark in the figure, $\mathrm{Ni}$ is a round blue line, $\mathrm{Co} @ \mathrm{Ni}$ is a yellow positive triangle line, and $\mathrm{Ni} @ \mathrm{Co}$ is a green inverted triangle line. d-g. Calculated electrostatic potential maps of Co-Ni Nanoclusters/rods growth system. $V s(\boldsymbol{r})$ values on the $0.01 e / \mathrm{Bohr}^{3}$ isodensity surfaces of the Co-Ni Nanoclusters/rods growth system. The most positive $V_{S, \max }$ values are found at the terminal sites, followed by cylindrical surfaces, which corresponds to the ordering of catalytic activity.

\subsection{Work function and charge transfer}

Then we studied the work function, which is also closely related to surface properties. The definition of work function is as follows:

$$
\Phi=E_{v a c}-E_{F}
$$

$\Phi$ is the work function, $E_{v a c}$ is the electrostatic potential of the vacuum level, and $E_{F}$ is the Fermi level. We calculated the work function in the Y-axis and Z-axis directions 
of Co-Ni nanoclusters/rods (Figure 3a) (The work function diagram of each structure is shown in Figure S3-S6 detailed work function value is shown in Table S3). When the nanoclusters grow into nanorods, the work function in the Z-axis direction is generally lower than that in the Y-axis direction (Figure 3a-3b, green arrows), which means that the surface of the nanorods in the Z-axis direction is more conducive to the electrons transfer between the catalyst and the electrolyte. Furthermore, the catalytic activity at terminal sites of the Z-axis direction is superior, which explains why the terminal $\sigma$ hole increases the activity of the catalyst from the perspective of the work function. In addition, we found that the nanorods' electrostatic potential with the Co-shell is generally lower than the Ni-shell (Figure 3a-3b). In short, in our energy research, Co atoms tend to be enriched in the rod core thermodynamically, $\mathrm{Ni}$ atoms tend to be enriched on the surface, so the nanorods with Ni surface exhibit a higher work function. Thus has relatively low activity, high stability surface. The nanorods with Co surface exhibit a lower work function and thus have a surface with high activity, low stability.

a
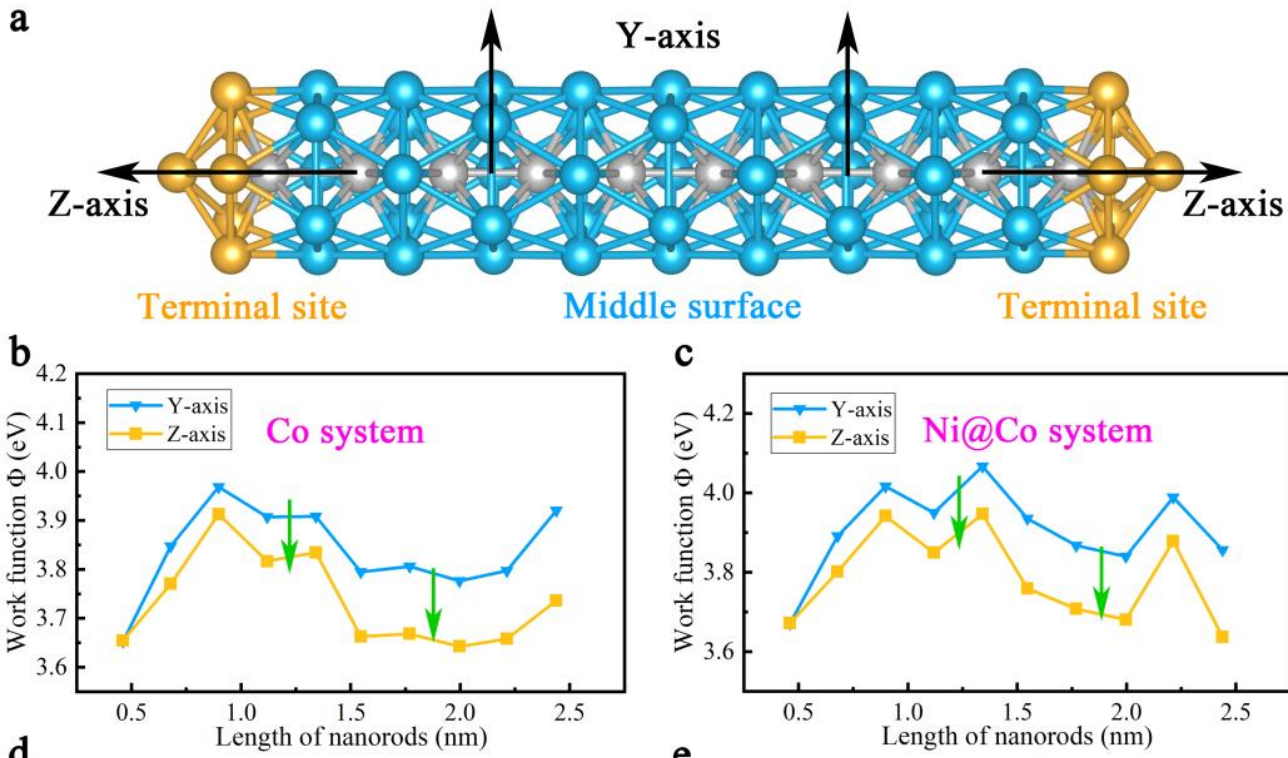

Middle surface

Terminal site
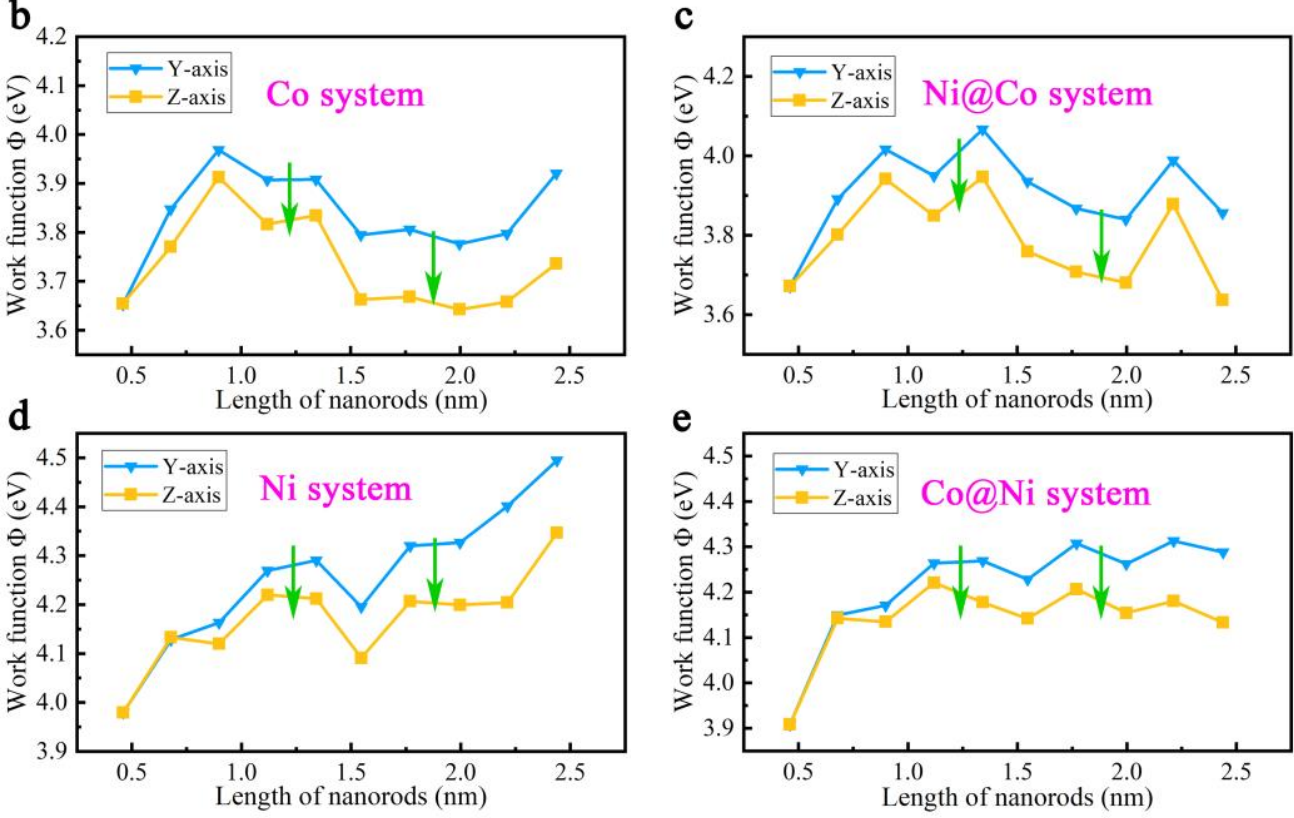
Figure 3. a. The work function in the Y-axis and Z-axis directions, the middle surface atoms (blue) and the terminal sites atoms (yellow) are in the Y-axis and Z-axis directions, respectively. b-e. The calculated work function of Co-Ni Nanoclusters/rods growth systems.

Although the work function can explain the formation of terminal $\sigma$-hole in the nanorods to a certain extent, it is beneficial to the electron transfer between the catalyst and the electrolyte, improving the catalytic performance. However, it is still unclear whether this improvement in electron transfer is related to the formation of terminal $\sigma$ hole or the charge distribution of the material itself. So we further carried out statistics on the charge distribution of the system. In addition, the status of charge transfer (or redistribution) in the systems is also one of the essential factors of its reactivity and catalytic activity. Therefore, we performed a Bader charge analysis on pure and alloyed $\mathrm{Co}$, Ni nanoclusters/rods. The Bader charge analysis $\operatorname{code}^{43}$ was utilized to calculate the overall atomic charge distribution and the difference in charge transfer between the core and shell of the nanoclusters/rods. The calculation formula of atomic charge difference can be expressed as:

$$
\Delta \rho=\rho_{\text {total }}-\rho_{\text {surface }}-\rho_{\text {core }}
$$

Where $\Delta \rho$ is the charge transfer difference, $\rho_{\text {total }}$ is the overall charge density, $\rho_{\text {core }}$ is the shell charge density of nanoclusters/rods, and $\rho_{\text {core }}$ is the core charge density.

Figure 4 shows the atomic charge distribution of the nanoclusters/rods. Due to the surface effect, we can observe that most of the nuclear atoms of pure $\mathrm{Co}$ and $\mathrm{Ni}$ nanoclusters/rods are slightly positively charged but basically in a neutral state. Interestingly, the nuclear atoms of the longer Co nanorods show a small number of negatively charged atoms. For the core-shell nanoclusters/rods structures, the Ni@Co system with Co on the surface presents a negatively charged core and a positively charged shell. The Co@Ni system with Ni on the surface presents a positively charged core and a negatively charged shell. This charge transfer (or redistribution) indicates 
that the difference in electronegativity between elements can be used to change the charge distribution of nanoclusters/rods by doping with other specific elements.

Two main factors dominate the charge transfer in these systems: the difference in element electronegativity and the surface effects. Due to the electronegativity difference between $\mathrm{Co}(1.88)$ and $\mathrm{Ni}$ (1.91), Bader charge analysis shows that the charge is always transferred from $\mathrm{Co}$ atom to $\mathrm{Ni}$ atom. At the same time, due to surface effects, electrons tend to transfer from the inside of the material to the surface. Therefore, pure Ni and Co@Ni core-shell nanoclusters/rods systems both have a positive core and a negative shell. The pure Ni system is due to the surface effect, while in the alloy phase of the Co@Ni system, the difference in electronegativity and the synergistic effect of the surface effects will further enhance the charge transfer. For pure $\mathrm{Co}$ and Ni@Co core-shell nanoclusters/rods systems, the surface effects have significant antagonism to electronegativity, but we found that the surface effects are weaker in the pure Co system, only shown in the shorter nanorods. Therefore, in the

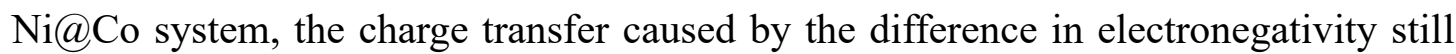
dominates, resulting in a negative core and a positive shell in the system.

In order to further study the cross-sectional charge transfer of high-length nanorods, we have calculated the charge density differences of periodic pseudo-one-dimensional nanorods (Figure S7a-S71). Interestingly, we found an electron-enriched ring between the core atoms and the shell atoms of the nanorods (as shown in Figure S7e-S7h), which means that Co-Ni nanowires may have good conductivity. Meanwhile, we found that the atomic charge transfer activity in the shell layer of the nanorods on the Ni surface is more intense than that on the Co surface (as shown in Figure S7i-S71).

According to the above analysis of the charge transfer (or redistribution), we have not observed the accumulation of positive or negative charges at the terminal sites in nanorods. Therefore, the nanorods' charge transfer (or redistribution) itself is not the direct cause of the reduction of the work function at the terminal sites and the 
improvement of the electron transfer between the catalyst and the electrolyte. The formation of terminal $\sigma$-hole is not simply caused by charge transfer (or redistribution).

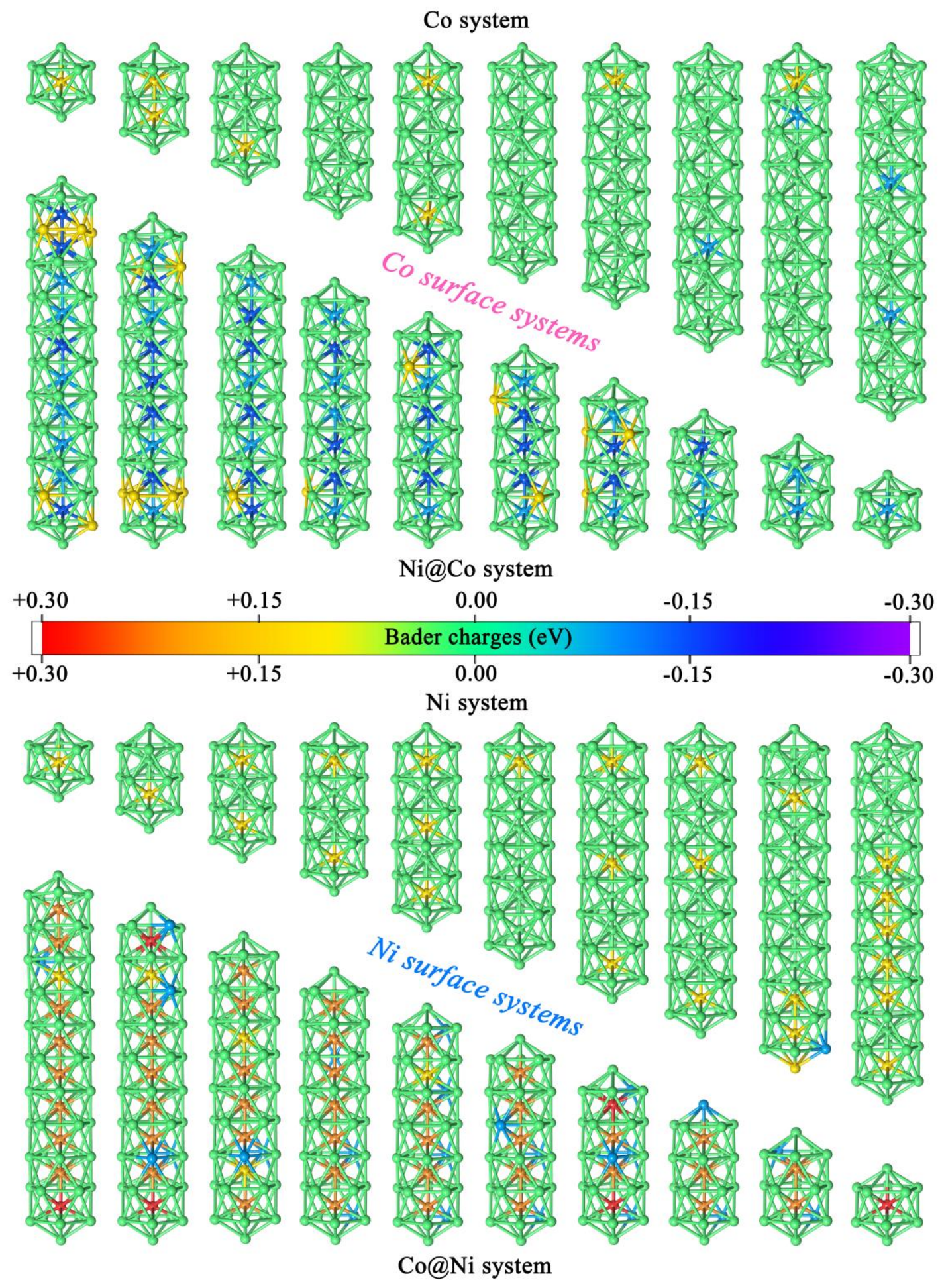

Figure 4. The Bader charge analysis of Co-Ni Nanoclusters/rods system. 


\section{5. d-band center and partial densities of states (PDOS)}

In order to further explore the relationship between terminal $\sigma$-hole and chemical activity, the change in reactivity and catalytic activity caused by the growth process of geometric structure and charge transfer, we further studied the electronic structure of the system, plotted the density of states of different orbitals of Co-Ni nanoclusters/rods, and calculated the d-band center. The calculation formula for the d-band center is as follows ${ }^{44}$ :

$$
\varepsilon_{d}=\int_{-\infty}^{+\infty} \rho_{d}(\varepsilon) \varepsilon d \varepsilon / \int_{-\infty}^{+\infty} \rho_{d}(\varepsilon) d \varepsilon
$$

Where $\varepsilon_{d}$ is the center value of the d-band, $\rho_{d}$ is the density of states of the d-orbital, and $\varepsilon$ is the energy width of the d-orbital. The d-band center near the Fermi level is often considered to have higher chemical activity ${ }^{45}$ (Figure 5a).

First, we perform d-band center analysis on all atoms in the system. We observe that for the pure Co system, except for nanoclusters, the trend of the d-band centers in the nanorods system is increasing (Figure 5b), attributed to the fact that the aggregation of the d-orbital partial densities of states (PDOS) in pure Co system at $E-E_{f}>0 \mathrm{eV}$. (Figure S8a, marked with a black dashed ellipse and a magenta arrow) The Ni@Co system, which also has a Co shell, is similar. (Figure S8c) For pure Ni and Co@Ni systems with a Ni shell, the all-atoms d-band center decreases with the growth of nanoclusters/rods. The main reason is that the electronic structure of the pure Ni and Co@Ni systems is different from that of the pure Co and Ni@Co systems. The d-orbital PDOS at $E-E_{f}>$ $0 \mathrm{eV}$ is not observed aggregation but aggregates at $E-E_{f} \quad<0 \mathrm{eV}$ (Figure S8b, S8d). We know that the Co-shell nanorods' overall chemical activity increases during the growth process while the Ni-shell nanorods are decreasing. In general, the order of the allatoms chemical activity of nanoclusters: $\mathrm{Ni}>\mathrm{Co} @ \mathrm{Ni}>\mathrm{Co}>\mathrm{Ni} @ \mathrm{Co}$, nanorods $(<2.5$ nm): Co@Ni > Ni > Co > Ni@Co. The PDOS of nanoclusters/rods $(<2.5 \mathrm{~nm})$ in 
different lengths and configurations are plotted in Figure S9. We found that the spin-up and spin-down curves of PDOS of all structures are asymmetric, which explains from the electronic structure that all structures are ferromagnetic. For the pure Co system (as shown in Figure S9a), after Co nanoclusters grow into nanorods, most of the nanorods spin-up d-band shifts to lower energy level, and spin-down d-band transfers to higher energy level. However, it is interesting that for the Co-10 nanorods about $2.5 \mathrm{~nm}$, the dband are all shifted to higher energy levels. In the pure Ni system (Figure S9b), with the growth of nanoclusters/rods, the d-band of most lengths of nanorods hardly shift, but Ni-9 and Ni-10 nanorods have been observed to have a large shape change in the dband. From the pure $\mathrm{Co}$ and $\mathrm{Ni}$ system, we found that the electronic structure will change significantly when the nanorods grow to a certain length. However, it is interesting that the PDOS curves of alloyed Ni@Co and Co@Ni nanoclusters/rods of different lengths are very similar, in which the d-band of the Co@Ni system almost overlap (Fig. S9d), indicating that after the nanorods alloyed, the d-band of the structure hardly shifts during the growth process, and the electronic structure became stabilization (Figure S9c, S9d).

Next, in order to explore the relationship between terminal $\sigma$-hole and chemical activity, we performed d-band center analysis on the atoms forming terminal $\sigma$-hole and other atoms in the middle positions (Figure 5c). As we expected, in almost all structures, the d-band center of the atom forming the terminal $\sigma$-hole is closer to the Fermi level than other atoms (Figure 5d-5g, green arrows), which means that the atoms formed the terminal $\sigma$-Hole exhibit higher chemical activity. Furthermore, we analyze the density of states of different orbitals in the longest nanorods of Co, Ni, Co@Ni, and Ni@Co systems. -T represents terminal atoms, -M represents middle atoms (all other atoms except terminal atoms). We find that the d-orbitals dominate the PDOS in all systems, and the terminal atoms' d-orbitals are shifted close to the Fermi level. Among them, the pure metal system is the most obvious. Both the spin-up and spin-down electronic density of states shift toward the Fermi level, which explains the theoretical reason for the increase in the chemical activity of terminal $\sigma$-hole from the perspective of the 
density of states.

In summary, through the PDOS calculation, we studied the changes in the reactivity and chemical activity caused by the growth process of the geometric structure and the charge transfer. Furthermore, we clarified the relationship between the terminal $\sigma$-hole and the chemical activity: the terminal atoms' d-orbital PDOS shifts toward the Fermi level and has a shallower d-band center than other atoms, thus exhibiting higher chemical activity. In addition, the catalytic reaction is often a multi-step complex process, and the chemical activity alone does not indicate the overall catalytic effect. According to previous reports, many catalytic processes (such as HER) require an appropriate $\varepsilon_{d}$ value to maintain the interaction strength between the reaction substrate and the catalytic site at an appropriate level ${ }^{44}$. Based on our calculation results, we found that the d-band center of Co-Ni nanorods changes regularly during the growth process, so we also provide researchers with a method for rationally designing catalysts. Researchers in different research directions can assist the d-band center's value in selecting the most suitable nanorods in length and composition.
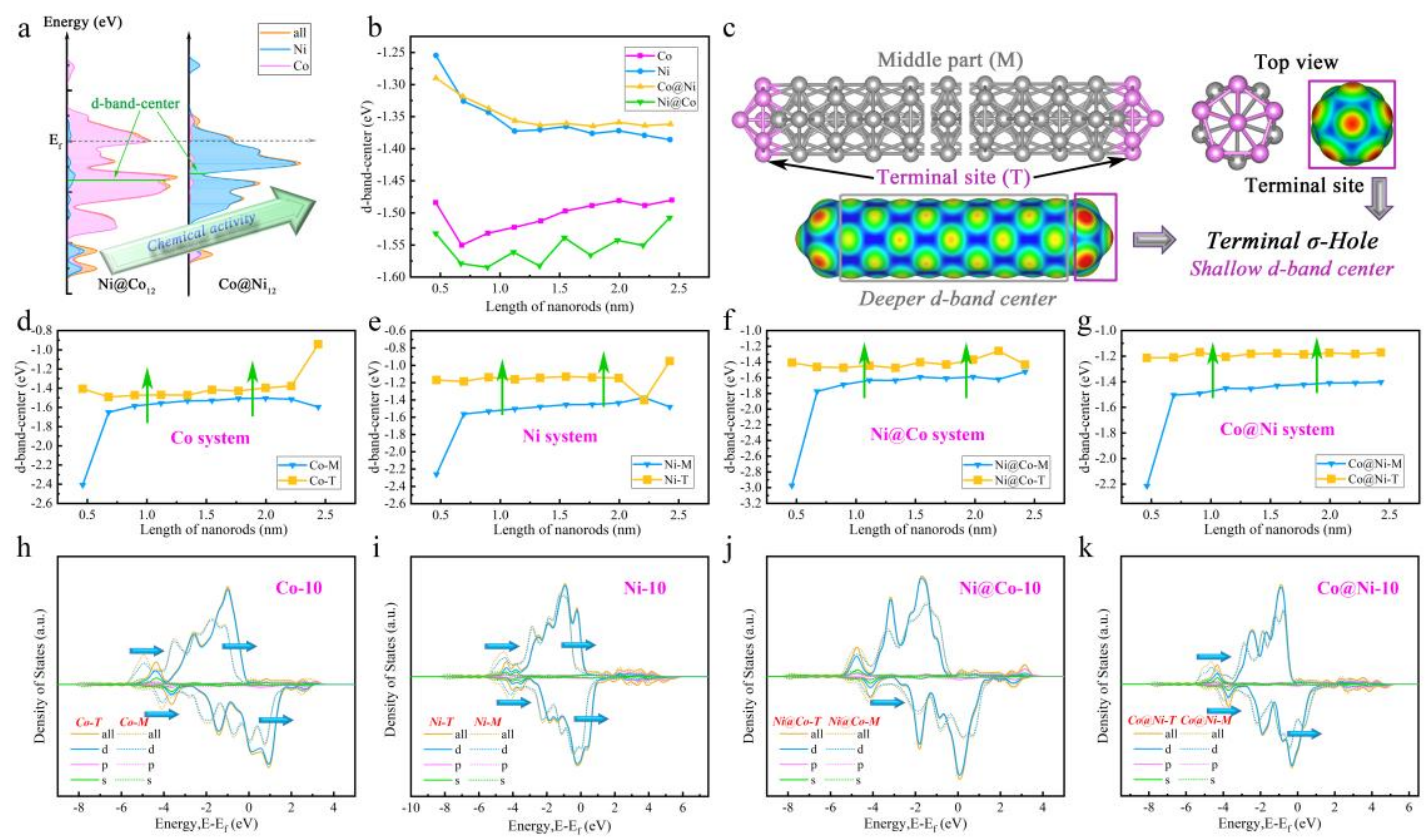

Figure 5. a. Schematic diagram of the d-band center. b. The d-band center value of all atoms during growth of Co-Ni Nanoclusters/rods system. c. The middle part (M) and 
Terminal site (T) of nanorods. Marked in gray and magenta, respectively. d-g. The dband center value of middle part (M) atoms and terminal site (T) atoms of nanorods. Middle part (M) atoms in blue and Terminal site (T) atoms in yellow. h-k. Calculated PDOS of the Co-Ni Nanorods. Fermi level is set at the zero of energy. The solid line represents the terminal sites atoms. The dotted line represents the middle part atoms. Among them, all orbitals are yellow lines, $\mathrm{d}$ orbitals are blue, $\mathrm{p}$ orbitals are magenta, and s-orbitals are green. The intensity of the density of states has been normalized.

\section{CONCLUSIONS}

In conclusion, we report the exceptional electrostatic phenomenon: terminal $\sigma$-hole in ultrathin nanorods, which not only provides a deeper theoretical understanding of nanorods catalysts but expands the $\sigma$-hole to nanorods for the first time. We conducted in-depth research on the geometric structure, cohesive energy, residual energy, magnetic moment, electrostatic potential, work function, charge distribution, and density of states during the growth of $\mathrm{Co}-\mathrm{Ni}$ nanorods through first-principle calculations. Our studies have shown that the terminal $\sigma$-hole sites exhibit low work function, shallow d-band center, and high chemical activity. The formation of terminal $\sigma$-hole is not simply caused by charge transfer (or redistribution), but the lowcoordination transition metals in nanorods' terminal sites remain additional semi-filled d-orbitals, causing the d-band shifts to the Fermi level, thus exhibiting a highly chemically active terminal $\sigma$-hole. It is the first time the terminal $\sigma$-hole phenomenon in nanorods has been reported, and to analyze the theoretical basis of terminal $\sigma$-hole from wide angles such as electrostatic potential, work function, charge distribution, and the density of states. Our research provides theoretical guidance for the prediction and characterization of the catalytic performance of nanorods systems.

Conflict of Interest: The authors declare that there are no conflicts of interest.

\section{SUPPORTING INFORMATION}


- See Supporting information for the Exceptional electrostatic phenomenon in ultrathin nanorods: the terminal $\sigma$-hole (PDF).

\section{ACKNOWLEDGMENTS}

This is a personal interest study without funding. We are grateful to Xu's personal workstations codenamed "Hydrogen" and "Lithium" for their high-performance calculation.

\section{REFERENCE}

1. Zheng, J. et al. Gold Nanorods: The Most Versatile Plasmonic Nanoparticles. Chem. Rev. 121, 13342-13453 (2021).

2. Guan, D. et al. Exceptionally Robust Face-Sharing Motifs Enable Efficient and Durable Water Oxidation. Adv. Mater. 33, e2103392 (2021).

3. Su, B., Cao, Z.-C. \& Shi, Z.-J. Exploration of earth-abundant transition metals ( $\mathrm{Fe}, \mathrm{Co}$, and $\mathrm{Ni}$ ) as catalysts in unreactive chemical bond activations. Acc. Chem. Res. 48, 886-896 (2015).

4. Tian, H., Li, X., Zeng, L. \& Gong, J. Recent advances on the design of group VIII base-metal catalysts with encapsulated structures. ACS Catal. 5, 4959-4977 (2015).

5. Ferrando, R., Jellinek, J. \& Johnston, R.L. Nanoalloys: from theory to applications of alloy clusters and nanoparticles. Chem. Rev. 108, 845-910 (2008).

6. Moroni, E., Kresse, G., Hafner, J. \& Furthmüller, J. Ultrasoft pseudopotentials applied to magnetic Fe, Co, and Ni: From atoms to solids. Phys. Rev. B 56, 15629 (1997).

7. Reck, R. \& Fry, D. Orbital and spin magnetization in fe-co, fe-ni, and ni-co. Phys. Rev. 184, 492 (1969).

8. Narayanan, T.N., Shaijumon, M.M., Ci, L., Ajayan, P.M. \& Anantharaman, M.R. On the growth mechanism of nickel and cobalt nanowires and comparison of their magnetic properties. Nano Res. 1, 465-473 (2008).

9. Hartmann, M.J., Millstone, J.E. \& Häkkinen, H. Surface Chemistry Controls Magnetism in Cobalt Nanoclusters. J. Phys. Chem. C 120, $20822-20827$ (2016).

10. Mao, X. et al. Computational high-throughput screening of alloy nanoclusters for electrocatalytic hydrogen evolution. NPJ Comput. Mater. 7, 46 (2021).

11. Nie, M. et al. Co-Ni nanowires supported on porous alumina as an electrocatalyst for the hydrogen evolution reaction. Electrochem. commun. 115, 106719 (2020).

12. $\mathrm{Xu}, \mathrm{H}$. Hydrogen evolution reaction on Co-Ni core-shell nanoclusters in different sizes: A DFT investigation from geometric structures to electronic 
structures. ChemRxiv (2021).

13. Niu, J., Ran, J., Qi, W., Ou, Z. \& He, W. Identification of active sites in CO2 activation on $\mathrm{MgO}$ supported Ni cluster. Int. J. Hydrog. Energy 45, 11108-11115 (2020).

14. Silaghi, M.-C., Comas-Vives, A. \& Copéret, C. CO2 Activation on Ni/ $\gamma-\mathrm{A} 12 \mathrm{O} 3$ Catalysts by First-Principles Calculations: From Ideal Surfaces to Supported Nanoparticles. ACS Catal. 6, 4501-4505 (2016).

15. Yao, S. et al. Exploring Metal-Support Interactions To Immobilize Subnanometer Co Clusters on $\gamma-\mathrm{Mo} 2 \mathrm{~N}$ : A Highly Selective and Stable Catalyst for CO2 Activation. ACS Catal. 9, 9087-9097 (2019).

16. Tu, W., Ghoussoub, M., Singh, C.V. \& Chin, Y.C. Consequences of Surface Oxophilicity of Ni, Ni-Co, and Co Clusters on Methane Activation. J. Am. Chem. Soc. 139, 6928-6945 (2017).

17. Breugst, M. \& Koenig, J.J. $\sigma$-Hole Interactions in Catalysis. Eur. J. Org. Chem. 2020, 5473-5487 (2020).

18. Lim, J.Y.C. \& Beer, P.D. Sigma-Hole Interactions in Anion Recognition. Chem 4, 731-783 (2018).

19. Koláŕ, M.H. \& Hobza, P. Computer Modeling of Halogen Bonds and Other $\sigma$ Hole Interactions. Chem. Rev. 116, 5155-5187 (2016).

20. Mallada, B. et al. Real-space imaging of anisotropic charge of sigma-hole by means of Kelvin probe force microscopy. Science 374, 863-867 (2021).

21. Clark, T., Hennemann, M., Murray, J.S. \& Politzer, P. Halogen bonding: the $\sigma-$ hole. J. Mol. Model. 13, 291-296 (2007).

22. Murray, J.S., Lane, P. \& Politzer, P. Expansion of the $\sigma$-hole concept. J. Mol. Model. 15, 723-729 (2009).

23. Stenlid, J., Johansson, A. \& Brinck, T. $\sigma$-Holes on Transition Metal Nanoclusters and Their Influence on the Local Lewis Acidity. Crystals 7, 222 (2017).

24. Stenlid, J.H. \& Brinck, T. Extending the sigma-Hole Concept to Metals: An Electrostatic Interpretation of the Effects of Nanostructure in Gold and Platinum Catalysis. J. Am. Chem. Soc. 139, 11012-11015 (2017).

25. Hvolbæk, B. et al. Catalytic activity of Au nanoparticles. Nano Today 2, 14-18 (2007).

26. Janssens, T.V. et al. Insights into the reactivity of supported Au nanoparticles: combining theory and experiments. Top. Catal. 44, 15-26 (2007).

27. Yu, Y., Cui, F., Sun, J. \& Yang, P. Atomic Structure of Ultrathin Gold Nanowires. Nano Lett. 16, 3078-3084 (2016).

28. Kresse, G. \& Furthmüller, J. Efficiency of ab-initio total energy calculations for metals and semiconductors using a plane-wave basis set. Comput. Mater. Sci. 6, 15-50 (1996).

29. Perdew, J.P., Burke, K. \& Ernzerhof, M. Generalized gradient approximation made simple. Phys. Rev. Lett. 77, 3865 (1996).

30. Kresse, G. \& Joubert, D. From ultrasoft pseudopotentials to the projector augmented-wave method. Phys. Rev. B 59, 1758 (1999). 
31. Blöchl, P.E. Projector augmented-wave method. Phys. Rev. B 50, 17953 (1994).

32. Sanville, E., Kenny, S.D., Smith, R. \& Henkelman, G. Improved grid-based algorithm for Bader charge allocation. J. Comput. Chem. 28, 899-908 (2007).

33. Henkelman, G., Arnaldsson, A. \& Jónsson, H. A fast and robust algorithm for Bader decomposition of charge density. Comput. Mater. Sci. 36, 354-360 (2006).

34. Rossi, G. et al. Magic polyicosahedral core-shell clusters. Phys. Rev. Lett. 93, 105503 (2004).

35. Baletto, F. \& Ferrando, R. Structural properties of nanoclusters: Energetic, thermodynamic, and kinetic effects. Rev. Mod. Phys. 77, 371 (2005).

36. Jena, P. \& Sun, Q. Super Atomic Clusters: Design Rules and Potential for Building Blocks of Materials. Chem. Rev. 118, 5755-5870 (2018).

37. Gao, L. et al. An artificial metalloenzyme for catalytic cancer-specific DNA cleavage and operando imaging. Science Advance 6, eabb1421 (2020).

38. Xu, H., Liu, P., Zhang, W., Wang, Q. \& Yang, Y. Structure, stability, electronic and magnetic properties of monometallic Pd, Pt, and bimetallic Pd-Pt core-shell nanoparticles. Chem. Phys. 539, 110953 (2020).

39. Andolina, C.M., Wright, J.G., Das, N. \& Saidi, W.A. Improved Al-Mg alloy surface segregation predictions with a machine learning atomistic potential. Phys. Rev. Mater. 5, 083804 (2021).

40. Rodríguez-López, J., Aguilera-Granja, F., Michaelian, K. \& Vega, A. Structure and magnetism of cobalt clusters. Phys. Rev. B 67, 174413 (2003).

41. Xu, X., Yin, S., Moro, R. \& de Heer, W.A. Magnetic moments and adiabatic magnetization of free cobalt clusters. Phys. Rev. Lett. 95, 237209 (2005).

42. Brinck, T., Murray, J.S. \& Politzer, P. Surface electrostatic potentials of halogenated methanes as indicators of directional intermolecular interactions. Int. J. Quantum Chem. 44, 57-64 (1992).

43. Tang, W., Sanville, E. \& Henkelman, G. A grid-based Bader analysis algorithm without lattice bias. J. Phys. Condens. Matter 21, 084204 (2009).

44. Wang, Y. et al. Probing the origin of group VB transition metal monocarbides for high-efficiency hydrogen evolution reaction: A DFT study. Appl. Surf. Sci. 539(2021).

45. Gates, B.C. \& Knozinger, H. Impact of surface science on catalysis. (2000). 